\title{
Assessing Cognitive and Behavioral Coping Strategies in Children
}

\author{
Gerly M. de Boo $\cdot$ Jelte M. Wicherts
}

Published online: 28 March 2007

(C) Springer Science+Business Media, LLC 2007

\begin{abstract}
The objective was to examine the psychometric qualities of a Dutch version of the Coping Strategies Checklist for Children (CCSC-R1) in the Netherlands and to replicate the 4-factor coping model that was established in an American population. Confirmatory factor analyses were used to investigate the factorial structure underlying the 13 subscales. A 5-factor model in which Positive Cognitive Restructuring was a separate coping dimension proved superior to a 4-factor model in which Positive Cognitive Restructuring was part of Direct Problem Solving. The 5-factor model proved invariant across age, and 10 subscales proved invariant across gender. Significant gender differences were found for the subscales "support for feelings," "distracting actions" and "wishful thinking," with girls scoring higher than boys. Pro-social behavior correlated positively with all five coping dimensions; the strongest correlations were with the coping dimensions Direct Problem Solving and Positive Cognitive Restructuring. Socially inadequate behavior was only significantly correlated with Avoidance Coping. The significance of coping assessment for cognitive and behavioral interventions for children with mental health problems was discussed.
\end{abstract}

Keywords Coping · Children · Cognitive strategies ·

Model fit · Factorial invariance

\section{Introduction}

The way children differ in their responses to stress is the subject of coping research. Stress is part of life and every child is exposed to stressful situations. Coping can be defined as the thoughts and behavior an individual uses to manage internal and external demands of situations that are appraised as stressful (Folkman \& Moskowitz, 2004). By

G. M. de Boo (凶) · J. M. Wicherts

Faculty of Social and Behavioural Sciences, Department of Clinical Psychology, University of Amsterdam, Roetersstraat 15, Amsterdam 1018 WB, The Netherlands e-mail: g.m.deboo@uva.nl 
studying individual differences in these stress reactions, researchers aim to clarify the relationship between stress and the development of mental health problems. This is not only of interest to the study of developmental psychopathology but also to clinical psychology because coping, when defined as effortful stress reactions (as opposed to reflexive or automatic reactions: Lazarus \& Folkman, 1984), can be subject to cognitivebehavioral interventions.

Coping is expected to moderate and mediate associations between stress and mental health problems (Compas, Connor-Smith, Saltzman, Thomsen, \& Wadsworth, 2001). Connor-Smith and Compas (2002) found that various coping strategies (e.g. primary control engagement, secondary control engagement, and disengagement coping) moderated the relationships between involuntary stress-reactivity, health status, and internalizing problems. Effective/adjusted coping were those reactions, which provided a buffering effect, whilst ineffective/maladjusted coping reactions put the child at risk for developing mental problems. Compas et al. (2001) reviewed empirical studies of the association between coping and psychological outcomes. They concluded that engagement coping and problem-focused coping were associated with better adjustment, and that disengagement coping and emotion-focused coping were associated with poorer psychological adjustment. However, these general findings were not conclusive and the quality of adjustment appeared to be related to the nature of the stressor being either controllable or not.

Coping is a complex multidimensional process that is sensitive to the demands and resources of the environment, to personality dispositions that influence the appraisal of the stressors and to the appraisal of the individual's available resources (Folkman \& Moskowitz, 2004). Children can appraise a stressful event as a challenge or a threat, either being controllable or uncontrollable. The outcome of coping affected coping efficacy beliefs, which in turn affected future coping efforts (Sandler, Tein, Mehta, Wolchik, \& Ayers, 2000). Coping efficacy mediated the relationship between active coping and psychological problems. In a longitudinal analysis Sandler et al. (2000) found that Active coping at Time 1 predicted increased Coping Efficacy at Time 2, and that Coping Efficacy at Time 2 mediated the relationship between Active Coping at Time 1 and internalizing problems. Coping mediated (together with negative appraisal) the relationship between multiple dimensions of control beliefs and mental health problems (Sandler, Kim-Bae, \& Mackinnon, 2000).

Folkman and Moscowitz (2004) argued that not only the nature of the stressor, but also characteristics of the individual influence the outcome of coping. For instance, Kochenderfer-Ladd and Skinner (2002) found that active coping in reaction to being bullied was beneficial for children who were not often bullied, but active coping was not beneficial for children who were persistent targets of bullying. They also found that avoidant coping, when being bullied, correlated positively with peer victimization for boys, but not for girls.

\section{Hierarchical structure of coping}

In the field of coping research there is lack of consensus concerning the operationalization of the construct "coping," and about the coping categories which best discriminate between children (Compas et al., 2001). Considering the complexity of the coping process, a theoretical framework could provide a structure to integrate the vast body of research. Skinner, Edge, Altman, and Sherwood (2003) have proposed a typology for classifying ways of coping. They distinguished one-dimensional lower order 
categories from multidimensional higher order categories and families of coping. Of the more than 1,000 different coping category systems reviewed by Skinner et al., only four category systems were empirically constructed, theory-based, and subjected to confirmatory statistical techniques with which the fits of multiple lower order ways of coping into higher order categories were tested. Confirmatory factor analysis is considered superior to exploratory factor analysis as it is less susceptible to the influence of chance. Moreover, confirmatory factor analysis can test the overall quality of the scales, as well as the relationship between scales. Furthermore, the tested coping model can be based on theoretical assumptions, whilst exploratory factor analyses are guided by intuitive and ad hoc rules (Kelloway, 1998). One of the category systems identified in the review by Skinner et al. (empirically constructed, theory-based, and tested by confirmatory statistical techniques) was developed by the research group of Ayers and Sandler.

Development of the children's coping strategies checklist

Ayers, Sandler, West, and Roosa (1996) have developed two measures of children's coping: How I Coped Under Pressure Scale (HICUPS) and Children's Coping Strategies Checklist (CCSC). ${ }^{1}$ The items of the HICUPS refer to a specific stressor that has happened in the past; the items of the CCSC refer to problems in general. In essence, the items of both self-report questionnaires are similar. The items of the HICUPS and the CCSC were derived from a content analysis of children's responses to a semi-structured interview combined with items from a review of the literature on coping during childhood and adolescence. A panel of students familiar with children's coping classified the items into 11 lower order categories with $80 \%$ agreement. In a revised version of the CCSC (CCSC-R1) four cognitive subscales were added aiming to broaden the dimension Positive Cognitive Restructuring (Ayers \& Sandler, 1999).

Factor structures of the CCSC

Ayers and colleagues analyzed various factor structures of the CCSC and the HICUPS (Ayers et al., 1996). At the basis of the hypothesized structures lay two theoretical models: the problem-focused versus emotion-focused model by Lazarus and Folkman (1984) and the approach versus avoidance focus model by Billings and Moos (1981) and Ebata and Moos (1991). A 5-factor model was tested, in which the 11 lower order categories were hypothesized to fit into 5 higher order categories: Problem Focused Strategies (including cognitive decision making and direct problem solving), Direct Emotion Focused Strategies (including seeking understanding, positive cognitive restructuring, and expression of feelings), Distraction Strategies (including physical release of emotions and distracting actions), Avoidance Strategies (including avoidance actions and cognitive avoidance), and Support Seeking strategies (including problemfocused support and emotion-focused support). The 5-factor model did not fit the data well. The Direct Emotion Focused Strategies dimension was eliminated because its correlation with Problem Focused Strategies was too strong. A 4-factor model, in which

\footnotetext{
${ }^{1}$ The CCSC is supposed to measure dispositional coping and asks the child to react to the question "when I have a problem......". The HICUPS presents the same statements to the child, but the statements are phrased in the past tense. In the HICUPS the child is asked to refer to a problem that has occurred in the last 3 months "when I had problem X....." Details about the development of the CCSC and HICUPS can be found in the manual (Ayers \& Sandler, 1999 September).
} 
Problem Focused Strategies and Direct Emotion Focused Strategies (including the subscales positive cognitive restructuring and seeking understanding) were combined to form an Active Coping Strategy factor, provided the best fit to the data. The common factor underlying the subscales indicated that each subscale was directly focused (behaviorally or cognitively) on dealing with the stressor. The superiority of this 4-factor model was replicated in a second independent sample. In addition, in both samples the 4-factor structure proved to be invariant across age and gender. Both 2-factor models (emotion- versus problem-focused coping and active versus passive coping) did not fit adequately. Similar findings of inadequate fits of 2-factor models were reported in a study of Connor-Smith, Compas, Wadsworth, Thomsen, and Saltzman (2000).

\section{Variations of the CCSC}

The most extensive version of the CCSC is the CCSC-R1, which contains 14 subscales, including a variation of cognitive coping strategies. This version was used flexibly by the research group, depending on the nature of the research questions and practical issues (Ayers \& Sandler, 1999). For instance, in the study by Sandler, Pitss, and Tein (1995), coping was assessed using a version of the CCSC-R1 containing three higher order categories: Problem Focused Coping, Avoidance, and Positive Cognitive Restructuring. The Positive Cognitive Restructuring contained the subscales positive thinking, control and optimism, whereas the subscales repression and wishful thinking were part of the Avoidance dimension. In another study by Sandler et al. (2000) subscales were grouped to assess two higher order categories Active and Avoidance Coping. During the process of scale development not all subscales of the CCSC-R1 survived the test of psychometric adequacy. In the 1996 study by Ayers et al. the subscale expression of feelings was eliminated because the internal consistency of this scale was too low. The subscale minimization cross-loaded with 4 factors, so also this subscale was excluded from the CCSC-R1 (see manual: Ayers \& Sandler, 1999).

The aim of this study

The aim of the present study was to examine the factor structure of the CCSC-R1 in a sample of normal Dutch children aged between 8 years and 13 years (the three final grades in Dutch primary education). This was partly a replication of the study by Ayers et al. (1996), but we used the CCSC-R1 version (containing the four cognitive subscales: positivity, control, optimism, and wishful thinking, see Table 1). In the Ayers et al. study, only one cognitive subscale (cognitive restructuring) was used. In line with the findings of Ayers et al. we hypothesized that a 4-factor model would fit the data well. In the 4-factor model of Ayers et al., Positive Cognitive Restructuring (subscales: positivity, control, and optimism) loads on the Active Coping dimension. The subscale wishful thinking was added to the Avoidance dimension. The two other dimensions, Distraction and Support Seeking, were equivalent to the dimensions in the Ayers et al. study. However, because the CCSC-R1 was extended to include additional indicators of cognitive coping, we examined also a 5-factor model, in which Positive Cognitive Restructuring was a separate dimension. The 4- and 5-factor models of the CCSC (Ayers et al. study) and the CCSC-R1 (this study) are shown in Table 1.

Cognitive Restructuring is a relatively new family of coping, referring to an individual's active attempts to change one's view of a stressful situation, in order to see the stressor in a more positive light (Skinner et al., 2003). From a developmental point 


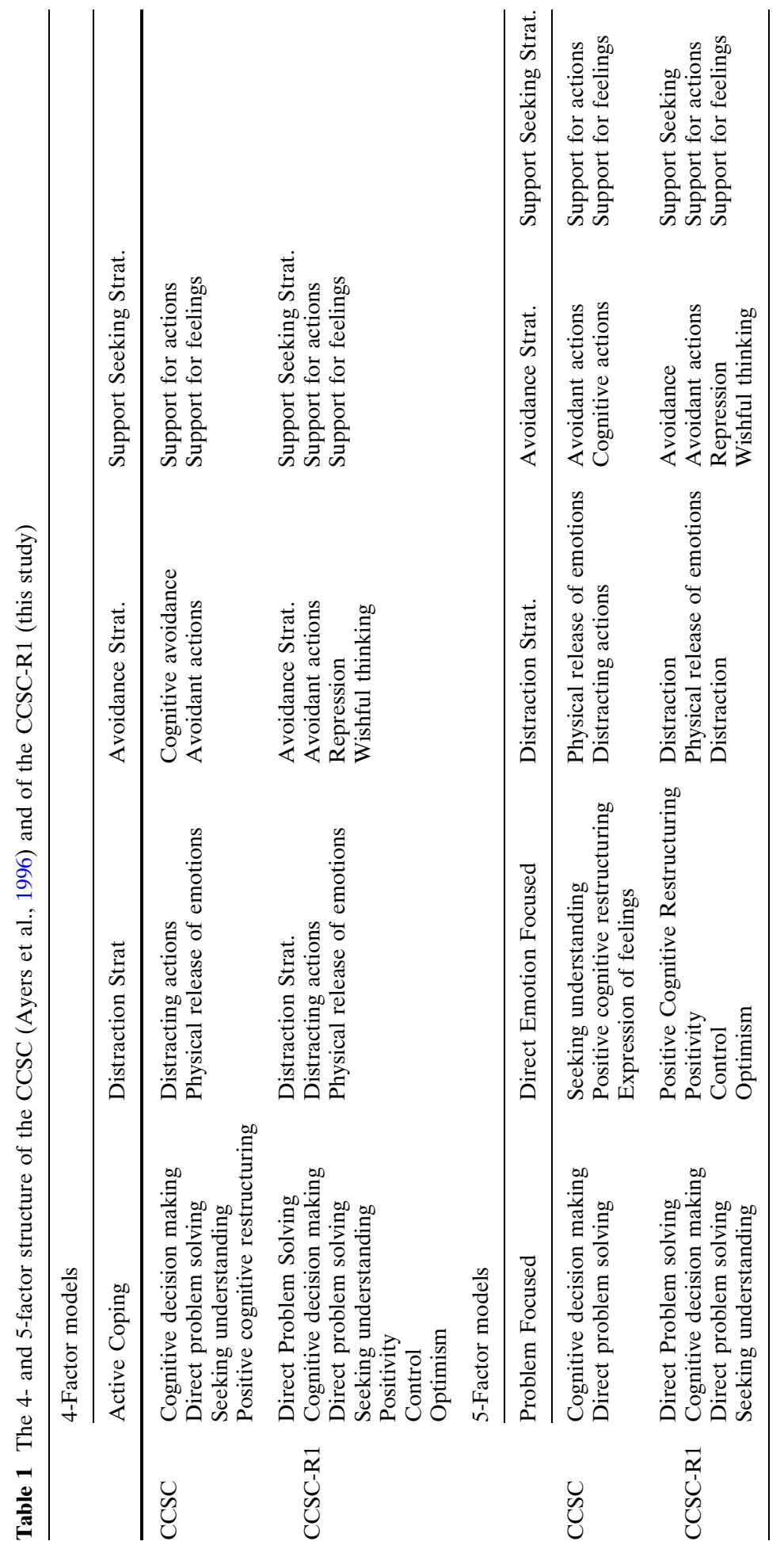


of view, a separate cognitive coping dimension can provide unique information. The later years of childhood can be characterized as a period of rapid cognitive, social, emotional, and physical change; it can be expected, therefore, that cognitive coping strategies will develop in a similar fashion (Forman, 1993). It will be of interest to clinicians and cognitive therapists to gain insight into these developmental aspects of cognitive coping (alongside behavioral coping) in this age group. Two-factor models were not fitted, because previous studies have found inadequate fits for these models (Ayers et al., 1996; Connor-Smith et al., 2000).

In addittion, we examined if the best fitting model was invariant across age and gender. Age and gender differences in coping have been reported. For instance Donaldson, Prinstein, Danovsky, and Spirito (2000) found that older adolescents (1518 years), when compared with younger children (9-14 years), tended to use a broader range of coping strategies. Donaldson et al. reported that emotion regulation increased with age. This was in line with previous findings of Rossman (1992) who found that younger children tended to use less emotion regulation strategies such as distraction and relaxation. On the other hand, Cognitive coping strategies, such as cognitive restructuring and decision-making, were found to increase in number and variety across primary school years (Ryan, 1989). With respect to gender differences, girls employed more emotion-focused strategies than boys (Donaldson et al., 2000). Hampel and Petermann (2005) reported that, in general, girls employed more maladaptive coping strategies and Hampel and Petermann found an interaction between age and gender. Compared with boys, girls showed a decrease in emotional regulating strategies and problem-focused coping and an increase in ruminating, resignation, and aggression. Interestingly, girls showed an increase also in support seeking, which was found to be the most robust difference in coping between adult men and women in the study by Tamres, Janicki, and Helgeson (2002). Tamres et al. found that women seek more emotional support. On the basis of previous findings (Donaldson et al., 2000; Rossman, 1992; Ryan, 1989) we hypothesized that older children would use more cognitive strategies, more emotion regulation, and would use more strategies overall than younger children. Furthermore, we expected girls to show less emotion regulation and less problem-solving, but to employ more support seeking coping (Hampel \& Petermann, 2005) than boys. Measures of coping need to be invariant across age and gender, however, if these differences are to be attributed to differences between individuals and not to item biases. In the current study, we examined measurement invariance of the factor structure of the CCSC-R1 across age and gender.

Furthermore, we studied the relationship between coping and adequate social functioning, which was operationalized in terms of adaptive and positive social behavior. The relationship between social behavior and coping has not been a subject of study, but coping has been studied in relationship to social competence (Kliewer \& Sandler, 1993; Reijntjes, Stegge, \& Meerum Terwogt, 2006). Social competence is a broader concept of social functioning and includes social behavior. Kliewer and Sandler considered social competence a particularly important variable, which affects children's coping responses. They argued that supportive social resources facilitated judging the appropriateness of behavior and helped children to regulate their emotions. It was expected, therefore, that social competence would affect the use of different coping efforts. Kliewer and Sandler found that children who scored higher on perceived social competence reported more active problem-oriented coping in response to stress. We hypothesized, in line with the above-mentioned studies, that there would be a positive 
relationship between adequate social behavior and the coping strategies Direct Problem Solving and Support Seeking.

\section{Method}

\section{Participants}

Four hundred and thirty-seven children (209 boys and 228 girls) completed the CCSC and 227 children also completed the MESSY (Matson, Rotatori, \& Helsel, 1983). The participants were enrolled in Grades 4-6 at eight different elementary schools (mean age $=10.5, \mathrm{SD}=1.0$ ); six schools were public elementary schools, two schools had a Christian background, three were inner-city schools, and five schools were situated in a village. Seventy-five percent of the children were of Dutch origin, $8 \%$ were of Moroccan origin, $2 \%$ of Turkish origin, $14 \%$ of the sample had mixed nationalities other than Dutch, Moroccan, Turkish, and $1 \%$ of the children did not provide information about their national or cultural background.

\section{Instruments/materials}

The CCSC-R1 version presented in the manual for the New Beginnings Project (Ayers \& Sandler, 1999) was translated and then translated back by two independent academics; one being the first author of this paper and the second a colleague specializing in Child and Adolescent Psychology. Agreement about discrepancies was reached following discussion. It was ensured that the meaning and phraseology of the original CCSC was retained in the Dutch version. The CCSC-R1 contained 54 statements which all start with "If I have a problem" followed, for example, by a statement such as "I tell others how I would like to solve it". The children could choose between four reactions: never (1), sometimes (2), often (3) and always (4). Scales, subscales, and examples of items are presented in Appendix A.

Social behavior was assessed by the Matson Evaluation of Social Skills for Youngsters (MESSY; Matson et al., 1983). In this study, we used the self-report version, which was appropriate for children aged 7-12 years. The scale contained 31 items, which described a wide range of positive and negative social behavior. Children rated each item on a 1-5 point Likert scale according to the frequency with which the behavior occurs or the child "felt like" the statement in the questionnaire $(1=$ not at all, $5=$ very much). The MESSY was translated and investigated in a Dutch population (Hulstein, 2005). A 2-factor model provided the best fit to the data $(n=1,416)$ (see Appendix B for scales and items). The two factors were labeled: "Pro-social" and "Socially Inadequate", comparable with the two primary factors identified by Matson et al. and by Spence and Liddle (1990). The Pro-social scale contained items in which the behavior of the person was beneficial to others; the Socially Inadequate scale contained social rule-breaking and inappropriate social actions. The internal consistencies (Cronbach's co-efficient alpha) of the Pro-social scale was .79 and of the Socially Inadequate scale .80 (Hulstein, 2005). Hulstein reported test-retest reliabilities (6weeks interval) of .70 for the Pro-social scale and .77 for the Socially Inadequate scale $(n=423)$. 
Procedure

For data collection various schools in the Netherlands were approached by phone and asked to participate. After receiving consent from the school's principal, letters and information were sent to all parents asking for passive consent for their child's cooperation. This procedure was approved by the Ethical Committee of the Clinical Psychology Department of our university under the following conditions: parents had to be fully informed, parents had the right to object at any time without restriction, and the school had to agree with this procedure. None of the parents objected. The CCSC was administered in the classroom and all children present at school that day participated. It took the children about 40 min to complete the CCSC and the MESSY. Children with reading difficulties were personally guided by the examiners. The children were free to ask questions if the items were unclear.

\section{Statistical analyses}

Several criteria were utilized to evaluate the psychometric characteristics of the CCSCR1. We examined the internal consistency of the subscales and the five higher order coping dimensions (i.e. Cronbach's alpha coefficient, mean inter-item correlations, range item-rest correlations).

Moreover, we tested various factor structures. Confirmatory factor analyses were conducted on the covariance matrices using LISREL 8.54 (Jöreskog \& Sörbom, 1993). Firstly, the factorial structure underlying the 13 CCSC subscales was investigated by fitting three models and comparing these in terms of model fit: a one-factor model (all items are best represented by one general coping factor), a 4-factor model in which three cognitive restructuring subscales were part of the Direct Problem Solving dimension, and a 5-factor model in which Positive Cognitive Restructuring (containing the three cognitive restructuring subscales) was a separate dimension (see Table 1). ${ }^{2}$ Secondly, the best fitting model was used to compare younger (8-10 years) and older children (11-13 years). Thirdly, the best fitting model was used to compare boys and girls. We investigated whether factor loadings, residual variances, measurement intercepts, and factor (co)variances were invariant across groups. The latter was done in a stepwise manner, which allowed for a test for each of these four types of constraints.

To assess the (relative) fit of the models, the following fit indices were used: $\chi^{2}$ with Degrees of Freedom (DF), Comparative Fit Index (CFI; Bentler, 1990), Root Mean Square Error of Approximation (RMSEA; Browne \& Cudeck, 1993), Standardized Root Mean Square Residual (SRMR), and the Expected Cross Validation Index (ECVI; Browne \& Cudeck, 1993). Hu and Bentler (1999) proposed that CFI values above .95, RMSEA values below .06, and SRMR values below .08 are indicative of good model fit. The ECVI is used to compare the relative fit of models. The ECVI is a measure of fit that takes into account the parsimony of the model, with lower values of ECVI indicating better fit.

The best fitting model was accepted for further analyses. Psychometric properties of the model were examined. By means of a MANOVA and a two-way between-subjects ANOVA, age and gender effects on coping were analyzed.

\footnotetext{
${ }^{2}$ We chose to perform the confirmatory factor analyses at the level of subscales, despite low reliability of some of the scales, because the sample sizes in the current study were too small to perform item level confirmatory factor analyses with polychoric covariance matrices and weighted least squared estimation. 
In a subpopulation of 227 children, the association between coping and social behavior (MESSY) was studied with Pearson correlations and regression analyses.

\section{Results}

Study sample

The results of seven subjects were excluded from statistical analyses because they missed too many items (i.e., more than 3 per questionnaire or more than 1 item per subscale). Other missing items were replaced by the mean item score of the total sample. Results of the coping indices (CCSC-R1) were analyzed for 430 children (47.4\% boys); the relationship between the CCSC-R1 and social indices (MESSY) were analyzed for a subgroup of 227 children (49.3\% boys). There was a significant age difference between boys and girls $(t=2.227, \mathrm{df}=428, p=.03,2$-tailed $)$ in the total sample. Boys were older $(M=10.6)$ than girls $(M=10.4)$, but in the mean difference was small. In the subgroup $(n=227)$ boys and girls did not differ significantly in age $(t=1.65, \mathrm{df}=225, p=.1,2$-tailed $)$.

\section{Model fit}

The upper part of Table 2 contains the fit indices of the three models. The 1-factor model showed poor fit. The values of RMSEA and SRMR of this model were considerably higher than the cut-off values for good fit. Moreover, the $\chi^{2}$ was very large and CFI value was clearly lower than the rule-of-thumb value of .95 . The 4 -factor model

Table 2 Fit indices of confirmatory factor analyses

\begin{tabular}{|c|c|c|c|c|c|c|c|}
\hline No. & Model & DF & $\chi^{2}$ & RMSEA & SRMR & CFI & ECVI \\
\hline \multicolumn{8}{|c|}{ Model fit of the CCSC } \\
\hline 1 & 1-Factormodel & 65 & 805.7 & 163 & .091 & .875 & 2.00 \\
\hline 2 & 4-Factormodel & 59 & 395.8 & .115 & .062 & .952 & 1.07 \\
\hline 3 & 5-Factormodel & 55 & 138.9 & .060 & .043 & .984 & .49 \\
\hline \multicolumn{8}{|c|}{ Equality constraints over gender } \\
\hline 4 & Configuration & 110 & 203.7 & .063 & .049 & .983 & .93 \\
\hline 5 & Factor loadings & 118 & 208.4 & .060 & .055 & .983 & .91 \\
\hline 6 & Residual variances & 131 & 227.2 & .059 & .056 & .982 & .89 \\
\hline 7 & Measurement intercepts & 139 & 270.9 & .067 & .059 & .975 & .96 \\
\hline \multirow[t]{2}{*}{8} & Free intercepts & & & & & & \\
\hline & disacsc* whisf** supfesc $* * *$ & 136 & 243.4 & .061 & .056 & .979 & .91 \\
\hline 9 & Factor (co)variances & 151 & 271.5 & .061 & .083 & .976 & .90 \\
\hline 10 & Free factor variance SSS $* * * *$ & 150 & 265.5 & .060 & .081 & .978 & .89 \\
\hline \multicolumn{8}{|c|}{ Equality constraints over age groups } \\
\hline 11 & Configuration & 110 & 218.2 & .068 & .052 & .980 & .97 \\
\hline 12 & Factor loadings & 118 & 225.7 & .065 & .058 & .980 & .95 \\
\hline 13 & Residual variances & 131 & 232.8 & .060 & .058 & .980 & .90 \\
\hline 14 & Measurement intercepts & 139 & 248.4 & .061 & .058 & .978 & .90 \\
\hline 15 & Factor (co)variances & 154 & 275.2 & .061 & .074 & .976 & .90 \\
\hline
\end{tabular}

Note: DF; Degrees of Freedom; RMSEA: Root Mean Square Error of Approximation; SRMR: Standardized Root Means Residual; CFI: Comparative Fit Index; ECVI: Expected Cross-Validation Index; * disacsc: Distraction; ** whisf: Wishful Thinking; *** supfesc: Support for feelings; **** SSS: Seeking Social Support. All p's $<.001$ 
showed a better fit than the 1-factor model, as evidenced by the lower ECVI value. The values of the CFI and the SRMR indicated that the 4-factor model fitted well. However, the $\chi^{2}$ was large, and the RMSEA was clearly above the cut-off value proposed by $\mathrm{Hu}$ and Bentler (1999). On the other hand, the 5-factor model showed a RMSEA value of .06. All fit indices clearly indicated that the 5-factor model was superior to the other models (see Fig. 1). Moreover in terms of RMSEA, SRMR, and CFI the 5-factor model showed good model fit.

\section{Invariance over gender}

To investigate whether the 5-factor model was invariant over gender, we fitted a series of increasingly restrictive models; the fit indices are reported in Table 2. The first step (i.e., No. 4) can be viewed as the baseline model. In this step only the configuration of the factor loadings was identical across both gender groups. That is, in this step the 5factor model was fitted in each gender group separately. As can be seen, this model again showed good model fit (although RMSEA was somewhat on the high side). In Step 5, the factor loadings were restricted to be equal across gender groups. This was accompanied by an improvement in RMSEA and ECVI. Moreover, because the CFI was not affected and because the chi-square increased only slightly (and nonsignificantly), this restriction of gender-invariant factor loadings appeared tenable.

In Step 6, the residual variances of each of the 13 subscales were restricted to be equal for boys and girls. Equal residual variances implied that, across groups, the

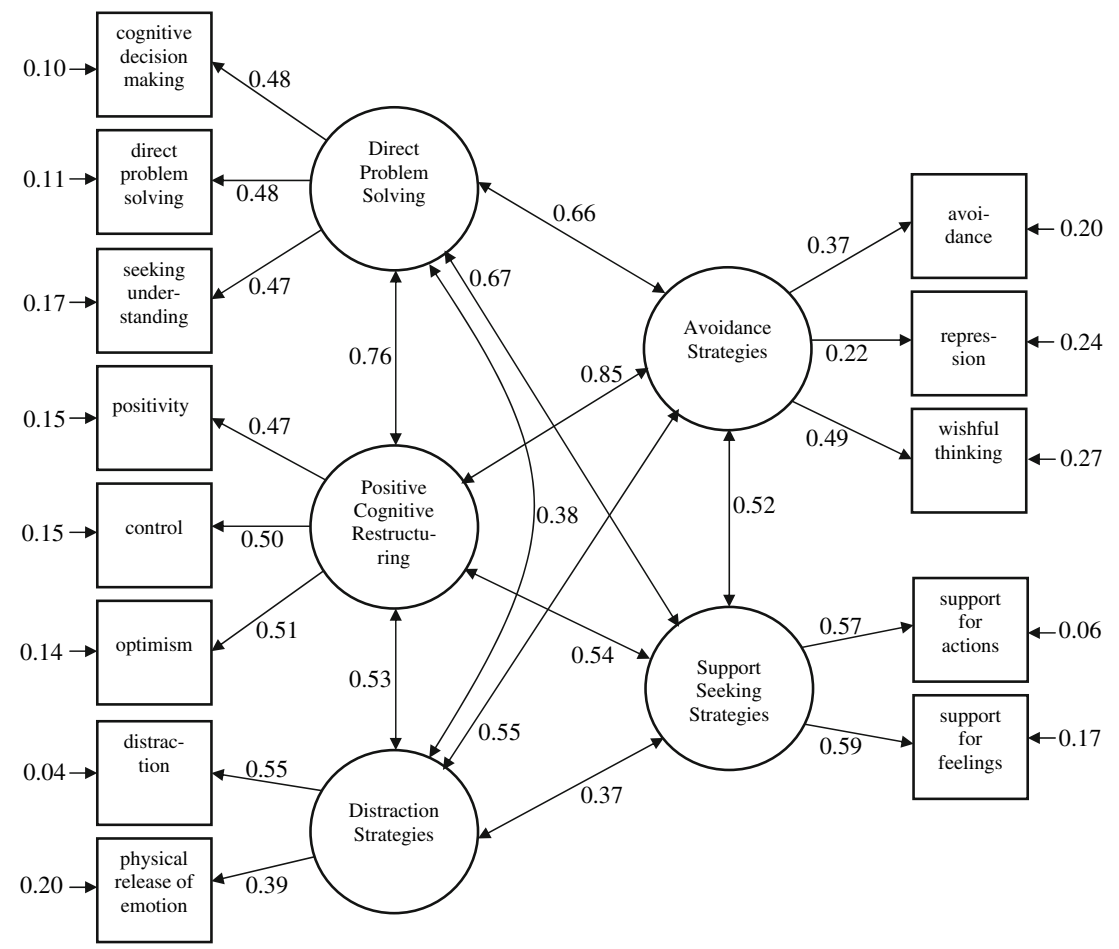

Fig. 1 Five factor model of the CCSC-R1

Springer 
subscale scores had the same amount of residual variance and measurement error (i.e., unreliability). Despite a small drop in CFI and a minute increase in SRMR, the fit indices indicated that this restriction was accompanied by an improvement in model fit. For instance, both the ECVI and the RMSEA of Step 6 were superior to those of Steps 5 and 4. Moreover, in light of the small (and non-significant) increase in chi-square, the residual variances appeared invariant over gender.

In Step 7, measurement intercepts were restricted to be invariant across gender. This restriction was a necessary condition for full measurement invariance across groups (Meredith, 1993). With this restriction, we tested whether group differences at the level of subscales could be explained by mean group differences in the latent factors underlying these subscale scores. A group difference in measurement intercept related to a subscale would imply the presence of measurement bias across groups. As can be seen, this restriction was accompanied by a deterioration of all fit indices. Hence, the overall restriction was not tenable, which indicated that mean gender differences on some subscales could not be due solely to gender mean differences at the level of the factors (i.e., there existed an unique mean gender difference on some subscales). Modification indices indicated that the misfit was related to the intercepts of the support for feelings, distraction actions, and wishful thinking subscales. In Step 8 we freed these three intercepts, which led to a considerable improvement of the model fit (cf. Step 8). In Step 8 none of the factor mean differences between males and females reached significance $(p<.05)$, indicating that the mean gender differences were absent at the level of factors.

In Step 9, variances of the five factors and the covariances between them were restricted to be equal across both gender groups. This restriction was accompanied by some model misfit in terms of SRMR and CFI. On the other hand, both the RMSEA and the ECVI indicated that this restriction was tenable. Modification indices indicated that the misfit was related to the variance of the Support Seeking Strategies factor. If this parameter was freely estimated over gender (Step 10), model fit improved considerably. The factor variance of Support Seeking Strategies was larger for girls. In conclusion, factor loadings, residual variances, and the majority of intercepts were gender-invariant. Apart from a small difference in factor variance of the Support Seeking Strategies factor, the factor variances and factor covariances were equal for gender. Gender differences in mean factor scores were not significant.

\section{Invariance over age}

The results of the tests of factorial invariance across younger ( $8-10$ years; $n=203)$ and older children (11-13 years; $n=227)$ are given in Table 2 . The fit measures in Step 11 indicate that the baseline model fitted sufficiently (although RMSEA was on the high side). In Step 12, factor loadings were restricted to be equal across age groups. This restriction was accompanied by a general improvement in model fit, indicating that the factor loadings were invariant over age groups. In addition, the fit measures in Step 13 indicated that residual variances were also invariant across age groups. Moreover, restriction of equal intercepts in Step 14 also appeared to be tenable. Hence, the CCSC$\mathrm{R} 1$ appeared to be fully measurement invariant with respect to age groups at the level of subscales. The factor mean differences between age groups were not significantly different from zero $(p<.05)$, which indicated that mean age group differences at the level of factors were absent. Finally, the factor variances and covariances between the five factors were restricted to be invariant across age groups in Step 15. Although the 
CFI and SRMR showed a slight worsening in model fit, the other fit measures indicated that this restriction was tenable. Therefore, differences in factor (co)variances between the two age groups were not found.

\section{Reliability}

The internal consistency coefficients for the subscales and the 5 dimension scales of the superior 5-factor model of the CCSC-R1 are presented in Table 3. In addition, Table 3 shows the homogeneity index and the range of the item-remainder correlations for all subscales and dimension scales. Cronbach's alpha for the five coping dimensions and six subscales (Seeking understanding, Control, Optimism, Wishful thinking, Support for feeling and Support for actions) were satisfactory (Cicchetti, 1994). Alphas between .60 and .70 were considered acceptable for research purposes because the mean inter-item correlation was also taken into account (Streiner, 2003). Cronbach's alpha for the subscales avoidant actions and repression were too low. However, considering the satisfactory homogeneity index and mean inter-item correlations, this could be attributed to the limited (only four) number of items in these subscales. In Table 3 internal consistency coefficients, homogeneity indexes and range of the item-remainder correlations were also presented for the MESSY. Both scales, Prosocial and Socially Inadequate, were psychometrically adequate for further analyses.

Table 3 Internal consistency (Cronbach's $\alpha$ ), homogeneity (mean interitem correlation), and range of item-remainder correlations of the CCSC-R1 and the MESSY

\begin{tabular}{llll}
\hline & $\begin{array}{l}\text { Cronbach's } \\
\alpha\end{array}$ & $\begin{array}{l}M \text { Interitem } \\
\text { Correlation }\end{array}$ & $\begin{array}{l}\text { Range Item-remainder } \\
\text { Correlation }\end{array}$ \\
\hline CCSC-R1 & & & \\
Problem Focused Coping & .85 & .33 & $.37-.64$ \\
$\quad$ Cognitive decision making & .64 & .31 & $.32-.48$ \\
$\quad .68$ & .35 & $.45-.47$ \\
$\quad$ Sirect problem solving & .68 & .38 & $.40-.58$ \\
Positive Cognitive Reframing & .71 & .34 & $.38-.65$ \\
$\quad$ Positivity & .66 & .33 & $.34-.52$ \\
$\quad$ Control & .75 & .43 & $.42-.62$ \\
$\quad$ Optimism & .70 & .36 & $.34-.55$ \\
Distraction Strategies & .77 & .28 & $.33-.54$ \\
$\quad$ Distracting actions & .62 & .25 & $.29-.49$ \\
$\quad$ Physical release of & .66 & .33 & $.43-.49$ \\
emotions & & & $.17-.53$ \\
Avoidance Strategies & .72 & .18 & $.25-.40$ \\
$\quad$ Avoidant actions & .51 & .21 & $.28-.33$ \\
$\quad$ Repression & .50 & .20 & $.45-.58$ \\
$\quad$ Wishful thinking & .72 & .39 & $.30-.75$ \\
Support Seeking Strategies & .88 & .44 & $.34-.62$ \\
$\quad$ Support for actions & .77 & .40 & $.58-.73$ \\
$\quad$ Support for feelings & .82 & .54 & $.28-.55$ \\
MESSY & & $.10-.52$ \\
Prosocial behavior & .79 & .21 & .18 \\
Socially inadequate behavior & .76 & .18 & \\
\hline
\end{tabular}

Note. CCSC-R1 = Children Coping Strategies Checklist, $N=430$; MESSY = Matson Evaluation of Social Skills (Dutch adaptation), $N=227$

Springer 
Analyses of age and gender differences of the CCSC-R1

In a two-way between-subjects MANOVA we analyzed the effects of age and gender on the five coping dimensions of the CCSC-R1. The multivariate main effect for age ( $F(25$, $1546)=1.187, p=.240)$, and the interaction between age and gender ( $F(15$, $1149)=1.015, p=$. 437) were non-significant. The multivariate main effect for gender was significant $(F(5,416)=2.872, p=.015)$, but the univariate main and interaction effects were not significant (all $p>.05$ ). In light of the previously detected intercept differences between the gender groups (see Step 7 of the confirmatory factor analyses) the multivariate main effect for gender on the level of these five dimensions was somewhat ambiguous. Therefore, we also ran a two-way MANOVA on the 13 subscales. This resulted in a non-significant multivariate main effect for age and a non-significant interaction effect between age and gender. The multivariate main effect for gender was significant $(F(13,408)=4.092, p<.001)$. The only univariate effects that reached significance were main effects for gender on the subscales distracting actions ( $F$ $(3,420)=10.524, p=.001)$, wishful thinking $(F(3,420)=3.996, p=.046)$, and support for feelings $(F(3,420)=6.984, p=.009)$. This was in line with the results from the factor analysis. On these three subscales, girls scored higher than boys (support for feelings: $M$ girls $=9.2 ; M$ boys $=8.4 ;$ Distracting Actions: $M$ girls $=11.8, M$ boys = 11.1; Wishful Thinking: $M$ girls $=9.96, M$ boys $=9.43$ ).

Analyses of age and gender differences of the MESSY

On the Pro-social and Socially Inadequate scales of the MESSY we found a significant main effect of gender, but not of age and no interaction effects. Girls scored higher on the Pro-social scale $(F(1,218)=6.612, p=.01)(M$ girls $=59.3 ; M$ boys $=57.7)$ and boys scored higher than girls on the Socially Inadequate scale $(F(1,218)=10.919$, $p=.001)(M$ boys $=34.2 ; M$ girls $=31.2)$.

Social behavior related to coping

It was hypothesized that socially adequate behavior would be positively related to coping strategies such as Problem Focused Coping and Support Seeking Strategies. In Table 4 the correlations between the five coping dimensions with the Pro-social and Socially Inadequate scale of the MESSY are presented. All dimensions correlated significantly and positively with the Pro-social scale. Only the Avoidant dimension was significantly correlated with the Socially Inadequate scale. In order to more fully

Table 4 Correlations between coping dimensions and pro-social and socially inadequate scales (MESSY)

\begin{tabular}{llllll}
\hline MESSY & \multicolumn{2}{l}{ Coping Dimensions } & & \\
\cline { 2 - 5 } & DPS & PCR & DS & AS & SSS \\
\hline Pro-social & $.45^{* *}$ & $.35^{* *}$ & $.2^{* *}$ & $.2^{* *}$ & $.33^{* *}$ \\
Socially Inadequate & -.11 & .07 & .13 & $.16^{*}$ & .06 \\
\hline
\end{tabular}

DPS (Direct Problem Solving), PCR (Positive Cognitive Reframing), DS (Distraction Strategies), AS (Avoidance Strategies), SSS (Support Seeking Strategies)

$* p<.05, * * p<.01$ 
Table 5 Summary of regression analysis for pro-social and socially inadequate scales predicting coping dimensions

\begin{tabular}{|c|c|c|c|c|c|c|c|}
\hline \multirow[t]{2}{*}{ Coping dimensions } & \multirow[t]{2}{*}{$R^{2}$} & \multicolumn{3}{|c|}{ Pro-social } & \multicolumn{3}{|c|}{ Socially inadequate } \\
\hline & & B & SE B & $\beta$ & B & SE B & $\beta$ \\
\hline DPS & .202 & .422 & .058 & $.443 * * *$ & ns & & \\
\hline PCR & .127 & .332 & .057 & $.367 * * *$ & . 109 & .054 & $.128^{*}$ \\
\hline DS & .066 & .147 & .042 & $.228 * *$ & .1 & .04 & $.165^{* *}$ \\
\hline AS & .079 & 187 & .051 & $.237 * * *$ & 145 & .048 & $.196^{* *}$ \\
\hline SSS & .121 & .278 & .051 & $.347 * * *$ & .089 & .048 & $.118^{*}$ \\
\hline
\end{tabular}

DPS (Direct Problem Solving), PCR (Positive Cognitive Reframing), DS (Distraction Strategies), AS (Avoidance Strategies), SSS (Support Seeking Strategies)

$* p<.05, * * p<.01, * * * p<.0005$

understand the predictive value of Pro-social and Socially Inadequate behavior we investigated the combined effects by using multiple regression analyses (enter method). The dependent variables were the five coping dimensions; the independent variables were the Pro-social and Socially Inadequate scales of the MESSY. Details of the Regression analyses can be found in Table 5. Direct Problem Solving was significantly predicted by Pro-social behavior $(F(2,224)=28.345, p<.0005)$, Adjusted $R$ square $=.195$. Positive Cognitive Restructuring was significantly predicted by Prosocial behavior and Socially Inadequate behavior $(F(2,224)=17.449, p<.0005)$, Adjusted $R^{2}=.127$. Distraction Strategies were predicted by Prosocial and Socially Inadequate behavior $(F(2,224)=7.943, p<.0005)$, Adjusted $R^{2}=.058$. Avoidant Strategies were predicted by Pro-social and Socially Inadequate behavior ( $F(2$, $224)=9.587, p<0005)$, Adjusted $R^{2}=.071$. Finally Support Seeking Strategies were predicted by Pro-social $(F(2,224)=15.346, p<0005)$, Adjusted $R^{2}=.113$.

\section{Discussion}

The aim of the present study was to cross validate a 4-factor structure of coping (in a sample of Dutch children) previously established by Ayers et al. (1996) in an American sample, and to explore if cognitive restructuring coping strategies could be represented by a separate factor in a 5-factor model. Our results indicated that the 5-factor model fitted the data best. This 5-factor model was proposed in the manual of the CCSC-R1, but had so far not been investigated. Skinner et al. (2003) identified the same five coping dimensions as the five most prevalent dimensions in their review. The additional fifth factor, labeled Positive Cognitive Restructuring, contained the subscales positivity, control and optimism. The aim of these coping strategies is to change one's view of a stressful situation in order to see the stressor in a more positive light (Skinner et al., 2003). The Ayers et al. 4-factor model contained only one cognitive restructuring subscale, which was fitted within the Problem Solving Strategies dimension. Two other studies have used confirmatory factor analyses to test conceptual models of coping. Cognitive strategies, however, were not represented by a separate factor in these models. Walker, Smith, Garber, and VanSlyke (1997) identified three factors: Active-, Passive-, and Accomodative coping. Behavioral and cognitive coping strategies were part of both Passive coping (acceptance) and Accomodative coping (distract/ignore and self-encouragement). Connor-Smith et al. (2000) distinguished three factors: primary 
control engagement coping, secondary control engagement coping, and disengagement coping. Cognitive coping strategies were part of all three factors in their model. They established fit indices of comparable strength to the fit indices of the 5-factor structure in this study.

Children's effortful stress reactions are a combination of cognitive and behavioral coping. The identification of a separate Positive Cognitive Restructuring dimension indicated that, within children's coping strategies, cognitive and behavioral coping could be distinguished. This finding enables researchers to study cognitive and behavioral strategies separately and their relationships to psychological symptoms. Therapists can also benefit from this finding: they can assess a child's inadequate cognitive strategies and direct interventions specifically towards the development of alternative and more adequate cognitive coping-provided they know what strategies are beneficial taking the type of stressor and the age of the child into consideration. More research will be necessary to clarify these issues.

The 5-factor model proved invariant for age. We could therefore use this model to analyse differences between older and younger children. We found no significant effect of age, whilst we expected older children to make more use of cognitive strategies and emotion regulation strategies. These age differences were reported by various authors (Donaldson et al., 2000; Rossman, 1992; Ryan, 1989). Possibly the age-range in this study was too small to detect age-related differences. Only two children were 8 years old and two were 13; the majority of children were aged between 9 and 12. In future studies the range should be extended into puberty. In the study by Donaldson et al. (2000) 914 year olds were compared with 15-18 year olds, and it was found that older children used a broader range of coping strategies and made less use of Distraction.

Girls did not make less use of Problem Solving than boys, which was contradictory to the outcome of the study by Hampel and Petermann (2005). However, some other gender differences were established. We found that girls employed more support for feelings than boys. This was also found by Hampel and Petermann and has also been reported by Tamres et al. (2002) in adult populations. Another difference found on subscale level was that girls (mean age in this sample 10.4 years) used more distracting actions and more wishful thinking than boys. Hampel and Petermann found that older girls (aged 12 and 13 years) used less distraction than younger girls, and they interpreted this finding as a decrease in adaptive coping strategies. Nolen Hoeksema and Girgus (1994) have argued that gender differences in coping before the onset of puberty play a role in the increased vulnerability of girls to develop depression in adolescence.

We studied the relationship between Pro-social behavior and coping. It was hypothesized that socially competent children would make more use of Active Problem-solving and Support Seeking. Our hypotheses were partly confirmed. Indeed children's Pro-social behavior was significant and positively correlated to the coping dimensions Direct Problem Solving, Positive Cognitive Restructuring and Social Support Seeking and less strongly correlated to Distraction and Avoidance. Pro-social behavior predicted $20 \%$ of the variance of the dimensions Direct Problem Solving; Socially Inadequate behavior proved not predictive. Kliewer and Sandler (1993) established also a significant positive association between problem-focused coping and social competence. They proposed that socially competent children had access to supportive social resources, which helped them regulate behavior and emotions. Children with socially inadequate behavior, which included antisocial behavior, suffered from rejection by their peers. Kliewer and Sandler hypothesized that these children were caught in a vicious circle. Inadequate social behavior led to stress in social 
relationships, which was not countered by adequate coping. This resulted in more rejection and more stress. This assumed process was partly underscored by the findings of Compas et al. (2001) and by the findings of this study. Compas et al. found that problem-focused coping was associated with fewer externalizing problems. We found a significant correlation between Socially Inadequate behavior and Avoidance coping. What was not expected was the correlation found between Avoidance coping and Prosocial behavior and the fact that all coping dimensions were significantly positively correlated to Pro-social behavior. Possibly this indicates that sociable children are flexible in their use of coping strategies and can perform a wide range of coping strategies. In this light, we also want to discuss the strong correlations among the different dimensions of coping. According to Connor-Smith et al. (2000) most individuals use multiple coping strategies, whilst higher levels of stress will result in increasing use of coping strategies of all types. Furthermore, Avoidant coping might not be inadequate as such, but its effects might be determined by the nature of the stressor as well as by the characteristics of an individual (Folkman \& Moscowitz, 2004). This was illustrated in the study by Kocherderfer-Ladd and Skinner (2002). Avoidance in reaction to peer conflicts was associated with less risk for developing anxiety for boys that were not frequently harassed and provided even some protective benefit for victimized boys. Coping strategies can not be judged either good or bad without information on the context, the nature of the stressor, and the characteristics of the coping individual.

A methodological limitation of this study is the exclusive use of self-report data. It is unclear whether a child's report accurately reflects what he does and thinks, or merely reflects what he would like to do and think. However, there is some evidence that children's self-reports are at least somewhat consistent with the way in which their peers view their coping efforts. Causey and Dubow (1992) found modest to moderate positive associations between self-report and peer ratings. Glyshaw, Cohen, and Towbes (1989) found moderate and significant correlations between mothers' reports of their adolescents' coping styles and the adolescents' self-report. Multi-informant information could shed more light on this issue. Unfortunately there is no parallel form of the CCSCR1 for other informants. Developing a multi-informant CCSC-R1 would enable future researchers to study this issue more closely. Another point concerns the fact that the CCSC-R and the MESSY are both paper-and-pencil self-report instruments. Shared method variance can have raised the association between coping and pro-social behavior. The fact that the correlations with the Socially Inadequate scale are mostly non-significant suggests, however, that shared method variance is not the primary source of the established correlations. Future research should include other methods of assessment. A positive feature of this study is the fact that the data came from a varied sample with no drop outs. Only the children who were not at school at the time of testing did not participate. The sample used in this study can therefore be considered fairly representative for the Dutch population. The established similarities in the structure of coping in Dutch and American samples indicate that coping is a reliable and stable construct representing ways in which children cope with stress. It is however possible that Dutch and American children share Western cultural and ethnical characteristics, and more research is necessary to shed light on possible ethnical and cultural differences in coping.

Compas et al. (2001) have argued that linking coping research with intervention research can prove fruitful in the prevention and treatment of psychopathology. Coping assessment can reveal strengths and weaknesses in a child's cognitive and behavioral

Springer 
repertoire when dealing with stress. In a clinical population, stress is an aspect of everyday life. Goals in cognitive behavioral therapy are behavioral changes in the direction of an active approach towards problems. Training skills are often part of behavioral interventions that are aimed to improve the child's coping abilities. Problemsolving skills aim to extend the child's response repertoire in difficult situations. Cognitive restructuring interventions aim to alter the child's irrational interpretations into more rational and less problematic thoughts, thus making room for changes in emotions and behavior. Another goal in therapy is to support the child's self-confidence, which can be reflected by a more active approach, and by positive cognitive restructuring. Part of a therapy with children is to influence their social context by teaching parents supportive and positive reinforcing attitudes. Therefore, it can be expected that the support seeking strategies of children change as a result of therapy. Coping assessment can provide essential pre-treatment diagnostic information as well as post-treatment evaluative information about the effects of interventions. Changes in coping strategies could be perceived as mediating mechanisms of change. These hypotheses can be subject of future research.

By translating an existing, theory-based, empirically tested coping scale for Dutchspeaking children, a step might be made in the standardization of coping measurement. The sound psychometric properties of the scale, and the invariant model fit across age, and (for 10 subscales) also across gender, open prospects for future studies to analyze developmental issues, gender differences, and intervention effects. The position of the construct "coping" on the interface between temperament, environmental stress factors, and mental health offers a unique angle to study the development of psychopathology. Finally, psychosocial interventions can be improved by a greater understanding of which coping strategies are beneficial for children with mental health problems.

\section{Appendix A}

Dimensions and subscales of the CCSC-R1 and examples of items

\section{Problem Focused Coping}

Cognitive Decision Making: You think about what you can do before you do something

Direct Problem Solving: You do something to make things better

Seeking Understanding: You try to figure out why things like this happen

Positive Cognitive Reframing

Positive Thinking: You remind yourself that you are better off than a lot of other kids

Optimistic Thinking: You tell yourself that you know what to do

Control: You tell yourself that you can handle the problem

Distraction Strategies

Physical Release of Emotions: You play sports

Distracting Actions: You listen to music

Avoidance Strategies

Avoidant Actions: You stay away from the problem

Repression: You just forget about it

Wishful Thinking: You imagine how you would like things to be 
Support Seeking Strategies

Support for Actions: You tell others how you like to solve the problem Support for Feelings: You tell people how you feel about the problem Source: Ayers \& Sandler, 1999 September

\section{Appendix B}

Items and subscales of the MESSY (Dutch adaptation)

Prosocial behavior scale

Makes other people laugh

Helps a friend who is hurt

Walks up to people and starts a conversation

Shows feelings

Says "thank you" and is happy when someone does something for him/her

Sticks up for friends

Looks at people when they are speaking

Smiles at people he/she knows

Works well in a team

Feels good if he/she help others

Asks questions when talking with others

Feels sorry when he/she hurts others

Joins in games with other children

Does nice things for others who are nice to him/her

Asks others how they are, what they have been doing etc.

Is friendly to new people he/she meets

Socially inadequate behavior scale

Threatens people or acts like a bully

Becomes angry easily

Gripes or complains often

Speaks (breaks in) when someone else is speaking

Takes or uses things that are not his/hers without permission

Slaps or hits when angry

Gives other children dirty looks

Picks out other children's faults/mistakes

Lies to get what he/she wants

Picks on people to make them angry

Makes sounds that bother others

Speaks too loudly

Gets into fights a lot

Hurts others when teasing them

Wants to get even with someone who hurts them

Source: Hulstein, 2005 


\section{References}

Ayers, T. A., \& Sandler, I. N. (1999, September). Manual for the Children's Coping Strategies Checklist \& How I Coped Under Pressure Scale. Retrieved June, 2006, from Arizona State Prevention Research Center Web site: http//www.asu.edu/clas/asuprc/.

Ayers, T. S., Sandler, I. N., West, S. G., \& Roosa, M. W. (1996). A dispositional and situational assessment of children's coping: Testing alternative models of coping. Journal of Personality, 64, 923-958.

Bentler, P. M. (1990). Comparative fit indexes in structural models. Psychological Bulletin, 107, 238-246.

Billings, A. G., \& Moos, R. H. (1981). The role of coping responses and social resources in attenuating the stress of life events. Journal of Behavioral Medicine, 4, 139-157.

Browne M. W., \& Cudeck, R. (1993). Alternative ways of assessing model fit. In K. A. Bollen \& S. J. Long (Eds.), Testing structural equation models (pp. 136-162). Newbury Park, CA: Sage.

Causey, D. L., \& Dubow, E. F. (1992). Development of a self-report coping measure for elementary school children. Journal of Clinical Child Psychology, 21, 47-59.

Cicchetti, D. V. (1994). Guidelines, criteria, and rules of thumb for evaluating normed and standardized assessment instruments in psychology. Psychological Assessment, 6, 284-290.

Compas, B. E., Connor-Smith, J. K., Saltzman, H., Thomsen, A. H., \& Wadsworth, M. E. (2001). Coping with stress during childhood and adolescence: Problems, progress, and potential in theory and research. Psychological Bulletin, 127, 87-127.

Connor-Smith, J. K., \& Compas, B. E. (2002). Vulnerability to social stress: Coping as a mediator or moderator of sociotropy and symptoms of anxiety and depression. Cognitive Therapy and Research, $26,39-55$.

Connor-Smith, J. K., Compas, B. E., Wadsworth, M. E., Thomsen, A. H., \& Saltzman, H. (2000). Responses to stress in adolescence: Measurement of coping and involuntary stress responses. Journal of Consulting and Clinical Psychology, 68, 976-992.

Donaldson, D., Prinstein, M. J., Danovsky, M., \& Spirito, A. (2000). Patterns of children's coping with life stress: Implications for clinicians. American Journal of Orthopsychiatry, 70, 351-359.

Ebata, A. T., \& Moos, R. H. (1991). Coping and adjustment in distressed and healthy adolescents. Journal of Applied and Developmental Psychology, 12, 33-54.

Folkman, S., \& Moskowitz, J. T. (2004). Coping: Pitfalls and promise. Annual Review Psychology, 55, $745-774$.

Forman, S. G. (1993). Coping skills interventions for children and adolescents. San Francisco, CA, US: Jossey-Bass.

Glyshaw, K., Cohen, L. H., \& Towbes, L. C. (1989). Coping strategies and psychological distress: Prospective analyses of early and middle adolescents. American Journal of Community Psychology, 17, 607-623.

Hampel, P., \& Petermann, F. (2005). Age and gender effects on coping in children and adolescents. Journal of Youth and Adolescence, 34, 73-83.

Hu, L. T., \& Bentler, P. M. (1999). Cutoff criteria for fit indexes in covariance structure analysis: Conventional criteria versus new alternatives. Structural Equation Modeling, 6, 1-55.

Hulstein, E. M. (2005). Construction and validation of a questionnaire to assess social skills in children and youth. Unpublished doctoral dissertation, University of Utrecht, the Netherlands.

Jöreskog, K., \& Sörbom, D. (1993). LISREL 8: Structural equation modeling with the SIMPLIS command language. Hillsdale, NJ: Erlbaum.

Kelloway, E. K. (1998). Using LISREL for structural equation modeling: A researcher's guide. Thousand Oaks: Sage Publications.

Kliewer, W., \& Sandler, I. N. (1993). Social competence and coping among children of divorce. American Journal of Orthopsychiatry, 63, 432-439.

Kochenderfer-Ladd, B., \& Skinner, K. (2002). Children's coping strategies: Moderators of the effects of peer victimization? Developmental Psychology, 38, 267-278.

Lazarus, R. S., \& Folkman, S. (1984). Stress, appraisal and coping. New York: Springer.

Matson, J. L., Rotatori, A. F., \& Helsel, W. J. (1983). Development of a rating scale to measure social skills in children: The Matson evaluation of social skills with youngsters (MESSY). Behavior Research and Therapy, 21, 335-340.

Meredith, W. (1993). Measurement invariance, factor analysis and factorial invariance. Psychometrika, $58,525-543$.

Nolen Hoeksema, S., \& Girgus, J. S. (1994). The emergence of gender differences in depression during adolescence. Psychological Bulletin, 115, 424-443. 
Reijntjes, A., Stegge, H., \& Meerum Terwogt, M. (2006). Children's coping with peer rejection: The role of depressive symptoms, social competence, and gender. Infant and Child Development, 15, 89-197.

Rossman, B. B. R. (1992). School-age children's perceptions of coping with distress: Strategies for emotion regulation and the moderation of adjustment. Journal of Child Psychology and Psychiatry, 33, 1373-1397.

Ryan, N. M. (1989). Identification of children's coping strategies from the school-agers' perspective. Research in Nursing and Health, 12, 111-122.

Sandler, I. N., Kim Bae, L. S., \& MacKinnon, D. (2000). Coping and negative appraisal as mediators between control beliefs and psychological symptoms in children of divorce. Journal of Clinical Child Psychology, 29, 336-347.

Sandler, I. N., Pitts, S. C., \& Tein, J.-Y. (1995, March). Positive cognitive restructuring in children of divorce. Paper presented at the Biennial Meeting of the Society for Research in Child Development, Indianapolis, IN.

Sandler, I. N., Tein, J.-Y., Mehta, P., Wolchik, S., \& Ayers, T. (2000). Coping efficacy and psychological problems of children of divorce. Child Development, 71, 1099-1118.

Skinner, E. A., Edge, K., Altman, J., \& Sherwood, H. (2003). Searching for the structure of coping: A review and critique of category systems for classifying ways of coping. Psychological Bulletin, 129, 216-269.

Spence, S. H., \& Liddle, B. (1990). Self-report measures of social competence for children: An evaluation of the Matson evaluation of social skills for youngsters and the list of social situation problems. Behavioral Assessment, 12, 317-336.

Streiner, D. L. (2003) Diagnosing tests: Using and misusing diagnostic and screening tests. Journal of Personality Assessment, 81, 209-219.

Tamres, L. K., Janicki, D., \& Helgeson, V. S. (2002). Sex differences in coping behavior: A meta-analytic review and an examination of relative coping. Personality and Social Psychology Review, 6, 2-30.

Walker, L. S., Smith, C. A., Garber, J., \& VanSlyke, D. A. (1997). Development and validation of the pain response inventory for children. Psychological Assessment, 9, 392-405. 